\title{
MODULI OF PARABOLIC $G$-BUNDLES
}

\author{
USHA N. BHOSLE
}

Introduction. Let $G$ be a connected reductive complex algebraic group. In this paper, the concept of parabolic structures on vector bundles [S, SM] is generalised to principal $G$-bundles. The relationship of parabolic bundles with unitary representations is studied and a coarse moduli space for semistable parabolic $G$-bundles is constructed using geometric invariant theory.

1. Let $T$ be a maximal torus of $G$ and let $L(T)$ be its Lie algebra. Let $L(G)$ denote the Lie algebra of $G$. Let $E$ be a holomorphic principal $G$ bundle on a compact connected Riemann surface $X$. Let $E(L(G))$ denote the bundle with fibre $L(G)$ associated to $E$ via the adjoint representation of $G$.

Definition 1.1. Fix points $x_{1}, \ldots, x_{n}$ in $X$. For each $i$, a parabolic structure on $E$ at $x_{i}$ is an element $\tau_{i} \in L(T)$ in $E(L(G))_{x_{i}}$. The bundle $E$ together with the parabolic structures $\left\{\tau_{i}\right\}_{i}$ is called a parabolic principal G-bundle.

DEFINITION 1.2. A parabolic $G$-bundle $E$ with parabolic structures $\left\{\tau_{i}\right\}_{i}$ is semistable (respectively stable) if for every reduction $\sigma: X \rightarrow E / P, P$ being a maximal parabolic subgroup of $G$, we have

$$
\text { degree } \sigma^{*}(T(G / P))+\sum_{i} \bar{\mu}\left(\bar{\tau}_{i}\right) \geq 0 \quad(\text { resp. }>0) ;
$$

where $T(G / P)$ denotes the tangent bundle along the fibres of $E / P \rightarrow X, \bar{\mu}$ is the form on the Lie algebra $L(P)$ of $P$ corresponding to the determinant of the adjoint action of $P$ on $L(G) / L(P)$ and $\bar{\tau}_{i}$ is the conjugate of $\tau_{i}$ lying in $L(P)$.

We remark that the notion of a reduction to a maximum parabolic is the correct generalisation of the notion of a subbundle of a vector bundle. Giving a reduction $\sigma$ as above is equivalent to giving a reduction of the structure group $G$ to a maximum parabolic $P$. When $G=G L(n, C), P=$ the isotropy subgroup of an $r$-dimensional subspace of $\mathbf{C}^{n}$, a $G$-bundle can be identified with a vector bundle $E$ and then giving a reduction of the structure group to the maximum parabolic $P$ is the same as giving a (proper) subbundle $F$ of $E$ of rank $r$ or to be more precise, an exact sequence $0 \rightarrow F \rightarrow E \rightarrow Q \rightarrow 0$. The inequality of Definition 1.2 then

Received by the editors July 19,1988 .

1980 Mathematics Subject Classification (1985 Revision). Primary 14F05; Secondary $14 \mathrm{D} 20$. 
translates as

$$
\begin{aligned}
0 \leq & (r \text { degree } E-n \text { degree } F) \\
& +\sum_{i}\left(r \text { weight } E_{x_{i}}-n \text { weight } F_{x_{i}}\right), \quad(\text { resp. }<0)
\end{aligned}
$$

This is just the definition of a parabolic semistable (resp. stable) vector bundle [SM]. In the absence of parabolic structure, the part $\sum_{i}(\ldots)$ in the above inequality is absent so that one gets Mumford's definition of a semistable (resp. stable) vector bundle. It can be checked that Definition 1.2 is independent of the choices involved.

1.3. EXAMPLE. We shall now give a construction of parabolic $G$-bundles using unitary representations generalising the constructions in [NS, §1, SM and $\S 6, \mathbf{A}]$. Let $\Gamma$ be a discrete subgroup of $\mathbf{P}(S L(2, \mathbf{R}))$ acting freely on the upper half plane $H$ such that $H / \Gamma$ has finite measure. Let $H^{+}=$ $H \cup$ parabolic cusps and $X=H^{+} / \Gamma$, a compact Riemann surface. For simplicity of exposition we assume that there is only one parabolic cusp, say $x_{0}$; the construction can be generalised easily for many cusps. Without loss of generality, $x_{0}$ can be taken as a point at infinity. Let $\gamma$ be the generator of the isotropy subgroup $\Gamma_{0}$ at $x_{0}$ given by $\gamma(z)=z+1$. For $\delta>0$, let $\bigcup_{\delta}=\{z=x+i y \mid y \geq \delta\}$, then $U=\bigcup_{\delta} / \Gamma_{0}$ is a neighbourhood of $x_{0}$ isomorphic to a disc minus a point. Let $\rho$ be a representation of $\Gamma$ in $G$ with $\rho(\gamma)=\exp \tau, \tau$ in $L(T)$. We associate to $\rho$ a parabolic $G$-bundle $E(\rho, \tau)$ with the parabolic structure $\tau$ at $x_{0}$ as follows. The bundle $E(\rho, \tau)$ is obtained by patching up $E_{\rho}=H \times{ }_{\rho} G$ on $X-x_{0} \approx H / \Gamma$ and the trivial bundle $U \times G$ on $U$ by the section of $E_{\rho} \mid U-x_{0}$ given by

$$
\psi_{\tau}: H \times_{\Gamma_{\infty}} \Gamma \rightarrow G, \quad \psi_{\tau}(z, a)=\rho(a)^{-1} \exp (-z \tau) .
$$

We show that if $\rho$ is unitary, $E(\rho, \tau)$ is semistable. If further, $\rho$ is irreducible, then $E(\rho, \tau)$ is stable. The construction can be generalised for more than one point easily.

1.4. Remarks. Semistable vector (or $G$-) bundles on a finite covering of a curve naturally give semistable parabolic vector (or $G$-) bundles on the base curve, e.g. (Proposition 1.2, 1.3 [B]). When the base curve is easier to study, this correspondence serves as a useful tool to study bundles on coverings. With a suitable definition of a parabolic structure, this is likely to be true for higher dimensional varieties too. Elementary transformations due to Maruyama and modifications of bundles over divisors will then give good examples of parabolic bundles. Another likely application will be in the study of desingularisations of moduli spaces of bundles as has been shown by Seshadri in [CS].

I have discovered recently that interesting results on the moduli of vector bundles on hyperelliptic curves in characteristic two and their relation with intersection of quadrics can be proved only by using parabolic bundles on $\mathbf{P}^{1}$.

In the spirit of Theorem 7.1 [A], Theorem 4.1 (2) [SM] and Theorem 2 [NS] we have the following theorem. 
THEOREM I. Let the genus of $X \geq 2$ and each $\tau \in T \cap[G, G]$. Then a holomorphic parabolic $G$-bundle on $X$ with a parabolic structure $\tau$ is stable if and only if it is of the form $E(\rho, \tau)$ for an irreducible unitary representation $\rho$ of $\Gamma$ with $\rho(\gamma)=\exp \tau$.

The proof of the theorem is lengthy and is on the lines of proofs in the above papers with many modifications.

2. THEOREM II. There exists a coarse moduli space $M$ for the equivalence classes of semistable parabolic G-bundles (of fixed topological type) on a compact Riemann surface $X$ with a fixed parabolic structure $\tau$ at $x_{0}$ in $X$, such that each $\tau \in T \cap[G, G] \cap K K$ being a maximal compact subgroup of $G$. The space $M$ is a normal complete variety. The subset $M^{s}$ of $M$ corresponding to stable parabolic G-bundles is a nonsingular open subvariety. One has $\operatorname{dim} M=\operatorname{dim} Z+(g-1) \operatorname{dim} G+\operatorname{dim} G / P$, where $Z=$ centre of $G, P=$ parabolic subgroup of $G$ determined by $\tau$.

SKETCH OF THE PROOF. Any construction of the moduli space of a class of bundles (using geometric invariant theory) consists of two main steps

(1) a construction of a universal space $R$ for the class of bundles,

(2) existence of a quotient of $R$ by a suitable group. For parabolic $G$ bundles, the step (1) is carried out similarly as in [AR]. Using the adjoint representation, the step (2) is reduced to proving the existence of a quotient of the universal space $R_{1}$ for parabolic Aut $L(G / Z)$-bundles by $S L(n)$. Let $L=L(G / Z), A=$ Aut $L$. A parabolic $A$-bundle can be regarded as a parabolic vector bundle $E$ (of $\operatorname{rank} k=\operatorname{dim}_{\mathrm{C}} L$ ) together with a Lie algebra structure on $E$. If $Y$ is the variety of Lie algebra structures on $\mathbf{C}^{k}$ isomorphic to that on $L$, then $Y \hookrightarrow \mathbf{P}=\mathbf{P}\left(L^{*} \otimes L^{*} \otimes L\right)$. Let $\Lambda$ denote the restriction of the generator of Pic $P$ to $Y$. One can choose an ample line bundle $B$ on $X$ such that $H^{0}(E \otimes B)$ is of constant dimension $n$ and it generates the fibre of $E \otimes B$ at every point of $X$ and for all semistable $E$. We choose $N$ points $x_{1}, \ldots, x_{N}$ of $X$. The fibres of $E \otimes B$ at $x_{i}$ determines a point of the Grassmannian $H_{n, k}$ of $k$-dimensional quotients of $\mathbf{C}^{n}$; further the Lie algebra structure on the fibre determines a point of the bundle $Q(Y)$ with fibre $Y$ associated to the universal quotient bundle $Q$ on $H_{n, k}$. Thus we get a morphism $f: R_{1} \rightarrow Q(Y)^{N}$. The parabolic structure gives a morphism $f_{2}: R_{1} \rightarrow H$ (p. 227, [SM]). Thus we have a morphism $f=f_{1} \times f_{2}: R_{1} \rightarrow Q(Y)^{N} \times H$ which can be seen to be injective for $N$ sufficiently large. Let $M_{1}$ be the ample line bundle $\sum_{i} L_{i}$ on $H_{n, k}^{N}$, $L_{i}$ being the generator of Pic $H_{n, k}$. Let $M_{2}$ be the ample line bundle on $H$ chosen in p. 227, [SM]. Define an ample line bundle $M_{3}$ on $Q(Y)^{N} \times H$ by $M_{3}=a k\left(M_{1}+M_{2}\right)+b\left(M_{2}+k \Lambda\right), a$ and $b$ being integers with $a \gg b$. This tricky choice of polarisations enables one to show (by explicit computation) that a point of $Z=Q(Y)^{N} \times H$ is semistable for $M_{3}$-linearisation iff its image in $H_{n, k}^{N} \times H$ is semistable for $\left(M_{1}+M_{2}\right)$-linearisation of the action of $S L(n)$. It then follows that the subset of $R_{1}^{s s}$ of $R_{1}$ corresponding to semistable bundles maps into $Z^{\text {ss }}$ (= set of semistable points of $Z$ ). Let 
$M$ denote the normalisation of the image of $R_{1}^{s s}$ in the geometric invariant theoretic quotient $Z^{s s} / S L(n)$. It is easy to show that $M$ is connected and so irreducible. By 1.3, there exists a morphism $p$ from the compact space $U$ of unitary representations to $M$ whose restriction $p_{0}$ gives an isomorphism from the open subset consisting of irreducible representations onto the nonempty open subset $M^{s}$ of $M$ corresponding to stable bundles (Theorem I). Thus the image of $p$ is complete and contains a nonempty dense open subset of an irreducible $M$, showing that Image of $p=M$. Thus $M$ is a complete normal variety. Now, as in [SM] we can show that $M$ is a categorical quotient and even a coarse moduli space.

\section{REFERENCES}

[A] A. Ramanathan, Stable principal bundles on a compact Riemann surface, Math. Ann. 213 (1975), 129-152.

[AR] _ Thesis, Tata Institute of Fundamental Research, Bombay, 1976.

[B] U. N. Bhosle, Degenerate symplectic and orthogonal bundles on $\mathbf{P}^{1}$, Math. Ann. 267 (1984), 347-364.

[NS] M. S. Narasimhan and C. S. Seshadri, Stable and unitary vector bundles on a compact Riemannian surface, Ann. of Math. 82 (1965), 540-567.

[S] C. S. Seshadri, Moduli of vector bundles with parabolic structures, Bull. Amer. Math. Soc. 83 (1977), 124-126.

[SC] _ Desingularisations of the moduli varieties of vector bundles on curves, Proc. Internat. Sympos. Algebraic Geometry, Kyoto, 1977, pp. 155-184.

[SM] C. S. Seshadri and V. B. Mehta, Moduli of vector bundles on curves with parabolic structures, Math. Ann. 248 (1980), 205-239.

School of Mathematics, Tata Institute of Fundamental Research, Homi Bhabha ROAD, BOMBAY-400 005, INDIA 